\title{
Evolución de ACT
}

\author{
Carmen Luciano \\ Universidad de Almería
}

RESUMEN

Se presenta la evolución de ACT a través de escenarios temporales que van surgiendo al hilo de la necesidad de mejorar y ampliar la comprensión del modelo, de los avances en la comprensión de sus componentes y de la investigación en lenguaje desde la Teoría del Marco Relacional. Primero, se presenta ACT en sus primeros años. Segundo, se presenta un escenario de proliferación de nuevos términos, datos y libros, al que continúa un escenario de reconsideración y abordaje para conectar ACT a las bases teóricas y experimentales del lenguaje y la cognición. Es este último escenario el que se ocupa de intentar una definición del repertorio problemático y del nuevo repertorio a partir de las transformaciones que ocurren al responder verbalmente a la propia conducta. Se concluye resaltando la coherencia de formular ACT como una terapia conectada al conocimiento experimental del lenguaje $y$, por tanto, coherente con la Contextual Behavioral Science, ámbito científico en el que ACT, como terapia contextual, es una parte de las aplicaciones de dicho conocimiento.

Palabras clave: Terapia de Aceptación y Compromiso (ACT); Evolución de ACT; Contextualismo Funcional; Teoría del Marco Relacional (TMR); Flexibilidad Psicológica; Ciencia contextual de la Conducta.
ABSTRACT

The evolution of ACT is presented through several temporal scenarios that emerge to improve the comprehension of the model and the ACT components. In addition, the research on language from the Relational Frame Theory begins to grow up.

The first scenario is ACT in the early years. A second scenario is characterized by the proliferation of new terms, books and data on the efficacy of ACT. Then, a renewed scenario showed up to relate ACT to the theoretical and experimental basis of language and cognition. The latest scenario involves the definition of the problematic repertory and the new repertory to be built up in terms of the transformations of functions that take place when responding or framing the own behavior in different ways. The formulation of ACT as a contextual therapy, explicitly connected to the experimental background on language, becomes coherent to the Contextual Behavioral Science, an overaching scientic model in which ACT, as contextual therapy, is one of its applications.

Keywords: Acceptance and Commithment Therapy (ACT); ACT Evolution; Functional Contextualism; Relational Frame Theory (RFT); Psychological Flexibility; Contextual Behavioral Science. 
La Terapia de Aceptación y Compromiso es una terapia de carácter contextual, funcional. Asume que los problemas de las personas están centrados en su historia personal en torno a cómo han aprendido no sólo a derivar pensamientos y emociones sino, lo más importante, a reaccionar a esos pensamientos y emociones. Dicho de otro modo, a qué función cumplen para hacer la vida o cómo, cuando se presentan, aprendemos a enmarcarlos para poder vivir. Los humanos entendemos reglas y formulamos nuevas reglas sobre la base de haber aprendido el tipo de comportamiento complejo que denominamos lenguaje. Aprendemos a seguir reglas sobre numerosos aspectos del entorno $y$, sin poder ser de otro modo, no sólo formulamos reglas sobre nosotros y lo que nos rodea sino que nuestra propia conducta, lo que sentimos y pensamos y las reglas sobre ello, llega a tener una función para conductas subsecuentes.

Por ejemplo, en un momento dado, escuchamos algo que nos hace sentir mal (tristeza, soledad,...). Ante ello hay dos opciones. Una, reaccionar a la función básica, primitiva, de la tristeza y, entonces, eludirla o escapar de ella de la forma en que se haya aprendido a hacer. En este contexto no habrá otra función presente que la de escape y la conducta sólo estará contextualizada por esa función. Al perpetuar este modo de actuar se formará un patrón psicológico en el que el escape/evitación predominan, tanto al buscar apresar lo que nos hace sentir bien como al alejarnos de lo contrario. Este tipo de regulación gira en torno a, o se limita, a conseguir, de inmediato, sentirnos bien, o mantener ese estado si ya lo hubiéramos al- canzado, lo que obliga a esquivar y mantener la atención en no sentir la tristeza o el malestar. Los resultados de este modo de organizar la vida no redundan en alcanzar una felicidad ( $y$ ¿cuál?), ya que cuando se presenta resulta efímera y se nos escapa. Además, no sólo fuerza nuestra atención a buscar dónde está sino a esquivar todo aquello que huela a lo contrario. $\mathrm{Si}$, en el corto plazo, la felicidad permanente es un bien imposible de alcanzar o mantener, en el largo plazo es destructivo. Produce numerosas pérdidas en forma de conductas que no se produjeron (p. ej., cosas que no hicimos...) porque suponían tener que contactar con la tristeza o la angustia, eventos que, en contra de lo establecido, son naturales a nuestra condición.

Sin embargo, la segunda opción para organizar la vida es no buscar una sensación de felicidad permanente sino dedicar los esfuerzos de cada minuto a vivir. En el fondo resulta banal y frívolo querer ser feliz en cada momento. No estamos hechos para eso y sí lo estamos para dar pasos en un proyecto de vida. Dicho de otro modo, es mejor dedicarse a vivir en paz que a buscar la felicidad y, su contrapartida, huir del malestar. En esa lógica, el modo de reaccionar al malestar, la tristeza, cuando se hacen presente, y se vistan como se vistan, es darles paso. Al hacerlo, lo estamos incluyendo como una parte de nosotros en ese momento $y$, por tanto, permitiendo que se hagan presentes otras funciones que puedan dar sentido a nuestro proceder.

En la primera opción habrá tristeza, angustia, encogimiento, atención centrada en ellas y poco más. En la segunda habrá apertura a sentir la angustia o la tristeza y otras sensaciones 
que se hagan presentes y, lo más importante, la apertura inclusiva permitirá contactar con funciones que, de acuerdo a la historia personal, harán que nos emocionemos, vibremos, que todo tenga sentido personal. Las acciones emprendidas en este contexto quedarán impregnadas de eso: de significado personal y podrá sentirse tranquilidad incluso con la angustia y la tristeza cerca. Pues bien, los problemas psicológicos están hechos de cómo aprendemos a organizar la vida, del tipo de repertorio generado a lo largo de nuestra historia personal en cuanto a qué hacer con la propia conducta que nos visita a cada paso (sean emociones, pensamientos, acciones). Es ahí donde se sitúa ACT.

En este artículo expongo los pilares de ACT como terapia contextual a partir de un recorrido breve de sus momentos evolutivos que, no obstante, siguen estando vigentes en la actualidad porque son formas útiles para muchas de las personas que conforman la órbita de las terapias psicológicas. Sin embargo, el consenso sobre la utilidad no está emparejado con la identificación de los procesos involucrados. Siempre habrá unos términos que resulten mejor para unos mientras otros términos lo serán para otros. Fuere como fuere, lo importante es avanzar en la comprensión de lo que hace que los cambios se produzcan. Es en esta línea donde la conducta del investigador cobra un papel principal y no está de más recordar los límites de sus conductas. Hacerlo podrá dar una perspectiva de la conducta científica y situará los cambios en los términos y en los modelos para afrontar el objeto de estudio correspondiente.

La conducta de investigar de los científicos, está sujeta a las mismas leyes que gobiernan el resto de conductas de los propios científicos y del resto de personas cuyo trabajo no sea hacer ciencia. Como Skinner dejó muy claro en 1974, hacer ciencia es sólo un modo especial de comportarse. El objetivo de esta conducta es el impacto que el producto científico genera en términos de capacidad de predicción y control, o capacidad de influir en los fenómenos de interés.

Desde este ángulo, la conducta del científico está sujeta a las fluctuaciones que rodean su propio trabajo y las funciones que estas fluctuaciones puedan tener en la conducta de cada científico harán que los productos científicos cambien a través del tiempo y el lector termine encontrándose ante un conglomerado de términos que pueden no ser funcionalmente equivalentes. Pero otras veces, representan ángulos en la temática que no habían sido considerados previamente y que sirven de insight, a modo de cambio de perspectiva que sitúa la investigación en otro plano. Sin poder ser de otro modo, el recorrido de ACT es el recorrido de los cambios en las conductas de quienes se han involucrado en su desarrollo.

Los pioneros señalaron el inicio de ACT allá por los años ochenta del siglo pasado y formularon el primer texto en 1999. A partir de ahí se ha producido una gran expansión, y en ello se continúa. Desde los primeros años hubo interés por los procesos básicos que gobernaban los componentes y las interacciones en ACT. Sin embargo, el foco en los nuevos términos, más genéricos (como el hexaflex y la inflexibilidad psicológica), sobre los componentes de ACT, se ha ido haciendo más fuerte y la expansión clínica y mediática ha sido tal que 
la voz del pequeño grupo de investigadores "básicos", centrados en lo molecular de los procesos clínicos, no tuvo la fuerza necesaria que algunos hubiéramos deseado. Ahora bien, en los últimos años, conjugar los componentes de la terapia a explicaciones básicas comienza a tener más volumen y parece que comienza un tiempo en el que habrá más espacio para esto. Siendo así, es el tiempo de mostrar con más precisión qué ocurre en las interacciones paciente-terapeuta, en cualesquiera de los momentos centrales en ACT. Por ello, me detendré en las "edades" de ACT a modo de tres escenarios vigentes que se cierran con la presentación de una terapia contextual centrada en los procesos relacionales involucrados.

\section{Escenario de la primera época:}

\section{a modo de la infancia de ACT}

En un capítulo publicado en 1987, una terapia con el nombre de Distanciamiento Comprehensivo hizo acto de presencia en lo que sería la forma precursora, en blanco y negro, de ACT (Hayes, 1987). El término no prosperó y el título de Terapia de Aceptación y Compromiso ACT- surgió con mayor fortuna. El primer texto completo se publicó en 1999 (Hayes, Stroshal, \& Wilson, 1999) y su planteamiento central en torno a la formulación de los casos y los elementos para afrontarlos es bien conocido: los problemas de las personas se centraban en torno a una clasificación funcional que se denominó trastorno de evitación experiencial (TEE) o evitación experiencial destructiva (EED) (Hayes et al., 1996; 1999; Wilson y Luciano, 2002). El TEE se definió como un modo de proceder adherido a la regla de "no estar dispuesto a es- tablecer contacto con las experiencias privadas molestas: estados o sensaciones, pensamientos y recuerdos molestos, problemáticos o negativos", y reaccionar fusionado a ello, lo que deriva, con frecuencia, en consecuencias de alivio y sensación de coherencia, de estar haciendo lo que se puede hacer. El problema se va alimentando de sucesivos ejemplos de evitación y conduce, a largo plazo, a la acumulación de pérdidas que empobrecen la vida, entristecen y producen un alto nivel de insatisfacción personal. Además, no resuelven el malestar sino que lo multiplican y lo extienden más allá de donde se inició.

Siguiendo su fundamento conductual, ACT concibió el TEE en términos de un seguimiento de reglas que resultaba problemático o limitante para la persona: una clase funcional de conducta que gira en torno al seguimiento generalizado de reglas donde priman las contingencias mediadas por otros o contingencias naturales a corto plazo pero problemáticas en el largo plazo. En uno u otro caso se trataría de personas que estarían siguiendo reglas para vivir que les impiden llegar a tener la vida que quisieran y les envuelven en la necesidad de seguir persiguiendo algo mientras, paradójicamente, se alejan de ello.

EI TEE se detectó como elemento común en trastornos diversos de acuerdo a los criterios taxonómico-burocráticos del DSM o la CIE. Así, funcionamientos depresivos, ansiosos, impulsivos, etcétera..., podían contemplarse unitariamente como patrones de TEE o EED. Los diferentes trastornos no serían sino modos diferentes de reaccionar para arreglar lo que se considera un problema: la presencia de pen- 
samientos depresivos, estados de ansiedad, la necesidad de consumir, las dudas, etcétera. Ante ello, reacciones de escape/evitación (podría ser discutir, rehuir, lesionarse, rumiar) tendrían las consecuencias esperadas pero no saldrían gratis. Por ejemplo, si se siente mucha rabia y la necesidad de consumir y se consume, la rabia y la necesidad se calmarían; si ante miedos al pasado o futuro se buscan explicaciones una y otra vez, dando vueltas y vueltas, con frecuencia conducen a callejones sin salida que llevan a la necesidad de acabar con estados de incertidumbre, malestar y cansancio. El propio enredo ante problemas que no tienen solución es el problema. Sin embargo, la coherencia de buscar solución mantiene a la persona en la búsqueda de soluciones y resulta en un círculo vicioso que no es gratis. Pasa factura en tanto que impide estar haciendo algo que realmente le hace a uno sentir que merece la pena seguir vivo. Las sucesivas reacciones de evitación, entre las cuales la rumia reina, hacen que la atención se reduzca a evitar el malestar, mientras escasa atención se sitúa en acciones que podrían llegar a emocionar y generar, antes o después, una sensación de paz o tranquilidad con uno mismo.

En la primera etapa de ACT se hablaba de alterar el patrón de evitación, generando otro patrón en el que primasen las funciones ligadas a acciones valiosas que dan sentido a la vida de cada uno, que merecen la pena. Y el patrón de evitación experiencial se aplicaba a cualquier tipo de problema, en tanto que los problemas, funcionalmente vistos, compartían el mismo fondo (no contactar con el malestar), aunque el modo en el que se presentara el ma- lestar y los modos de reaccionar para sacarlo de la escena fueran múltiples y diferentes de una persona a otra, hasta abarcar el extenso catálogo de trastornos.

Los textos iniciales de ACT se centraron en construir un nuevo repertorio en el que la persona pudiera reaccionar de un modo flexible a la presencia del malestar. Los procesos para abordar el tratamiento implicaban el análisis funcional del patrón de funcionamiento para que la persona pudiera contactar con el problema, el plan a seguir para arreglarlo y los resultados a corto y a largo plazo en su vida. Implicaba que la persona pudiera experimentar un estado de desesperanza al contactar con lo que desea, lo que hace y lo que consigue. Y desde ahí, ir abriendo el horizonte para vislumbrar el verdadero problema y su solución. Entre las fases de actuación terapéutica se haIlaban hacer experimentar que el control es el problema e interactuar para abrir direcciones con significado personal; la necesidad de enseñar a poder relacionarse con pensamientos y emociones de un modo más eficaz que redundase en una vida con más sentido personal. Ahí estarían representados los componentes de desliteralización, defusión y diferenciación de las dimensiones del yo.

El escenario inicial supuso una apertura de la terapia a muchos profesionales y muy diversos en su modo de concebir la naturaleza humana y en su conocimiento del análisis funcional de la conducta. Esta apertura fue inevitable y la necesidad de generar productos ajustados a las diferentes problemáticas se evidenció al mismo ritmo que se iniciaban los ensayos controlados $y$, muy especialmente, con el inicio 
de investigaciones para aislar el impacto de enmarcar la propia conducta (pensamientos, emociones, recuerdos) en oposición con las acciones valiosas, con la vida, versus hacerlo en inclusión (revisión en Ruiz, 2010). El "barco" de ACT se estaba haciendo más grande $y$, con ello, sus necesidades. El enorme interés que suscitaba se confrontaba con las críticas desde otros ámbitos de la psicología en general y la terapia en particular. Y no parece que pudiera ser de otro modo. Este contexto condujo a un nuevo escenario que denomino la época de la exuberancia.

\section{La época de la exuberancia de ACT: a modo de adolescencia}

Esta época se identifica por varias características. Se inicia una expansión de textos "especializados" dirigidos a diferentes problemáticas. Recordemos que ACT utiliza como modelo de psicopatología la regulación problemática de evitación experiencial. Entender este patrón en términos conductistas, como una clase funcional, como una operante relacional, quizás hubiera hecho innecesaria la proliferación de textos que ofrecían modalidades distintas en la misma dirección. En todo caso, los ejemplos facilitaban la comprensión y eso merecía la pena. A partir de 2003 y 2004 se dispara la publicación de textos que, de un modo u otro, mostraban el elemento común como "diferentes reacciones que, funcionalmente, estaban contextualizadas en términos de evitación del malestar", ya viniera éste vestido como dolor, ansiedad, depresión, impulsos de, etcétera.

Ahora bien, era evidente que el término de evitación experiencial hacía explícita la lucha contra el malestar mientras que los elementos de ACT podían ser aplicados en contextos no clínicos en los que las personas no experimentaban ninguna lucha especialmente relevante contra el malestar ni mostraban los costes de un patrón sistemático de evitación experiencial. Se hacía necesario un término más inclusivo que el de evitación experiencial, y el concepto de in-flexibilidad psicológica emergió para albergar un patrón de regulación que podía, o no, mostrar cronicidad y que podía estar directamente controlado por ya fueren funciones aversivas o apetitivas sin que las funciones relevantes de orden superior, o con significado personal, pudieran hacerse presentes.

Generar flexibilidad psicológica se convirtió en el modo de organizar ACT en la mayoría de los textos. En la misma lógica, los elementos iniciales de ACT quedaron condensados en lo que se conoció como el hexaflex; un hexágono cuyos vértices mostrarían los seis focos de intervención interconectados (contacto con el momento presente, aceptación, defusión, Yocontexto, Valores, y Acciones con significado) con el objetivo de generar flexibilidad. Se invocaba el mindfulness y la aceptación a la par que la identificación de los procesos de cambio centrados en el compromiso y la acción (Hayes, Strosahl, Bunting, Twohig, \& Wilson, 2004).

Los vértices de hexaflex no se formularon para representar procesos distintos pero, con frecuencia, no se entendió de modo que la búsqueda de un engranaje básico, centrado en el análisis de los componentes diferenciales entre vértices, se tornó una empresa casi imposible. El hexaflex ha conocido sucesivos in- 
tentos por simplificar sus vértices. Por ejemplo, como tres elementos (Abierto-Consciente-Implicado) por Stroshal (2012); como dos componentes (valores y defusion) en Luciano, Valverde y Gutiérrez (2004); como la matrix (Polk \& Schoendorff, 2014).

Otra de las características del escenario de la exuberancia ha sido la proliferación de ensayos controlados unidos a un control experimental mejor. En este sentido, el número de ensayos controlados de ACT supera el centenar y este hecho abrió la puerta a los tratamientos empíricamente validados, por ejemplo, en los ámbitos del dolor y de la depresión.

Y algo más a resaltar en este periodo. El desarrollo de la Teoría del Marco Relacional (TMR) (Hayes, Barnes-Holmes, \& Roche, 2001), como teoría que sustenta ACT y cuyo foco es el análisis funcional del lenguaje y la cognición, abrió las puertas al análisis de los métodos e interacciones clínicas en ACT. En este sentido, el período de exuberancia no sólo ha tenido como foco la proliferación del hexaflex, sino que también ha supuesto un gran avance en las investigaciones desde la TMR que, siendo minoritarias y difíciles de abordar, han comenzado a aislar los tipos de transformaciones responsables de algunos de los cambios involucrados en las interacciones clínicas de ACT y a dotar a la terapia de un marco explicativo básico.

Es el caso del texto de Niklas Törneke (2010) y es el caso de la investigación analítica funcional de las interacciones de defusión y de las dimensiones del yo desde los supuestos de la TMR. Estas investigaciones se han centrado en el análisis de las transformaciones que ocurren al enmarcar la propia conducta a nivel deíctico y jerárquico (yo-aquí-ahora y mi conducta ahí y perteneciente a mí) lo que actualiza la presencia de las funciones importante para uno y las acciones vinculadas a ellas (Luciano et al., 2011; Foody, BarnesHolmes, Barnes-Holmes, \& Luciano, 2013; Gil, Ruiz, Valdivia, \& Suárez-Falcón, 2015; López \& Luciano, 2015). Tipos de transformaciones que están presentes en la mayor parte de las interacciones/métodos de ACT y otras terapias contextuales.

\section{En resumen. Luces y sombras en la evolución de ACT y abriendo el paso}

La evolución de ACT ha generado luces y sombras, como sucede con cualquier empresa científica de gran dificultad.

Entre las sombras, cabría destacar las dificultades generadas por el modelo hexaflex para investigar los procesos involucrados en la terapia. Cierto es que el hexaflex no estaba comprometido con un análisis diferencial de sus vértices, en tanto que su incorporación en ACT tuvo más de términos medios, a modo de puentes, que de procesos independientes a nivel de la TMR. Fuera como fuese, la simpatía por el hexaflex en ACT puede haber desviado la atención de los investigadores tratando de buscar, en tales términos, los procesos de cambio y puede haber infravalorado el interés por el conocimiento básico de los componentes clínicos efectivos.

Entre las luces cabría destacar la extensión de ACT al hilo del término más inclusivo, la inflexibilidad, $y$, con ello, la facilidad de su comprensión a través de profesionales e 
investigadores con diferentes concepciones de la conducta. Y destacan, entre las luces, los ensayos controlados y, en un ámbito más modesto pero molecular, las investigaciones dirigidas al aislamiento de las condiciones que enredan a las personas y no les permiten vivir y aquéllas involucradas en que las personas se desembaracen del enredo.

Todo el engranaje generado en torno a ACT durante años representa un contexto motivacional relevante que en los últimos tiempos ha permitido dar un paso hacia delante, salir de la órbita del hexaflex y comenzar a centrar la atención, de modo más amplio, en los procesos básicos de cambio, en aislar las transformaciones involucradas en los problemas y en su solución. La ausencia de investigación sistemática sobre el análisis de los componentes responsables del cambio clínico ha provocado importantes críticas y puede conducir a que ACT sea una terapia sin suficiente apoyo empírico y experimental. Sin dicho soporte, ACT sería como una rama sin apenas sujeción al tronco básico del conocimiento científico sobre el comportamiento. Sus posibilidades de supervivencia serían limitadas.

\section{La época inclusiva: a modo de adultez}

A partir de 2012 el hexaflex recibe críticas explícitas debido a las dificultades en detectar los supuestos procesos diferenciales de sus componentes (por ejemplo, Foody, BarnesHolmes, \& Barnes-Holmes, 2012). Como ya señalé anteriormente, aunque los vértices del hexaflex se formularon como términos medios para facilitar la comprensión y la comunicación fue casi inevitable que se asumieran otros significados. En esa línea, se buscaron las conexiones entre cada punto del hexaflex y procesos verbales diferenciales según la teoría que lo sustentaba, la TMR. Sin embargo, esas conexiones diferenciales no se detectaron y sí lo que de algún modo está contemplado en el hexaflex: que sus componentes o vértices giran en torno a la flexibilidad psicológica y se yuxtaponen.

Es en estos tiempos cuando se ve fortalecido el incipiente contexto de investigación de las transformaciones involucradas en los componentes de ACT, especialmente la defusión. Ante tales evidencias, algunos investigadores nos lanzamos a buscar abiertamente el modo de centrar el modelo de ACT en términos del comportamiento relacional. Y fruto de ese proceso, iniciado en abril de 2012, es tanto el capítulo recientemente publicado por Törneke, Luciano, BarnesHolmes y Bond (2015), como el texto genérico publicado por Villate, Villate y Hayes (2015).

Como resumen de este proceso, cabría concluir que estaríamos ante una terapia que no se sustenta en lo graciosos, lógicos, oportunos o comprensibles que puedan resultar sus métodos y filosofía, sino en el aislamiento de los elementos/interacciones básicos que influyen en, o cambian, los problemas. Esto requiere una redefinición de los problemas y de las soluciones clásicas en ACT y, en esta línea, en Törneke et al., (2015) ofrecimos una formulación desde la TMR.

Los humanos aprenden a seguir reglas e interactuar con la conducta propia, y la conjugación de ambos elementos produce 
un patrón de funcionamiento en torno a la propia conducta eficaz o ineficaz. Este último caso sería el de la inflexibilidad psicológica y vendría a sustituir a conceptos previos, menos inclusivos, como augmenting ineficaz y evitación experiencial destructiva.

El patrón inflexible está hecho de conjuntos de acciones sin dirección valiosa, acciones directamente bajo control de la única función presente, la que emana del pensamiento/ emoción que esté en cada momento. Acción fusionada a tales funciones, lo que impide que las funciones ligadas a lo que tiene significado para la persona se hagan presentes $y$, por tanto, no es posible que la persona actúe bajo su control. La acumulación de acciones fusionadas a lo largo del tiempo resultará destructiva.

En términos relacionales, la inflexibilidad psicológica estaría hecha de múltiples ejemplos en los que la persona habría reaccionado "ciega" al enmarcar los pensamientos/ emociones que fluyen en equivalencia/ coordinación con uno mismo. Esto impide discriminar a uno mismo como diferente de la conducta que está produciéndose $\mathrm{y}$, por tanto, facilita permanecer "ciego" al resto de funciones que podrían hacerse presentes, pues se reacciona exclusivamente bajo control de las funciones que emergen con los pensamientos/emociones que hayan podido adquirir o derivarse por la historia personal. Metafóricamente, el problema son acciones entrampadas.

La flexibilidad es un modo especial de abordar la propia conducta y, en términos no técnicos, se entiende como la habilidad para observar y reaccionar a los pensamientos, sensacio- nes, acciones y tener la oportunidad de actuar hacia metas con sentido personal.

Al igual que la inflexibilidad, la flexibilidad también implica enmarcar la propia conducta pero de modo distinto. En el último caso sería enmarcar la propia conducta en perspectiva e inclusión, lo que permite la transformación de la propia conducta de un modo que resulta en acciones efectivas para vivir.

Mientras la inflexibilidad nos ciega a las funciones relevantes por la historia personal, la flexibilidad nos abre las puertas a tales funciones al requerir diferenciar la propia conducta de uno de uno mismo, como observador de ella. Al hacerlo, se hacen presentes las funciones ligadas a esa perspectiva, a la jerarquía inclusiva de uno con las propias conductas en un momento dado (Luciano, Valdivia, \& Ruiz, 2012).

En términos técnicos, hemos definido la flexibilidad psicológica como una clase funcional, una operante, que consiste en responder a la propia conducta en un marco de jerarquía con la perspectiva deíctica-YO. Esto permitirá (1) una reducción substancial en el control conductual de las funciones presentes al enmarcar en coordinación la propia conducta, sin distinguirla de uno mismo, y (2) un incremento de respuestas desde el enmarque jerárquico. Lo que significa dar la oportunidad para que se hagan presentes las funciones apetitivas de augmentals $y$, consecuentemente, las acciones coordinadas con esa clase relacional (Törneke, Luciano, Barnes-Holmes, \& Bond, 2015).

A nuestro modo de ver, el objetivo de la terapia contextual en virtud del grado de conocimientos actuales sería dar la oportunidad 
para construir el repertorio de flexibilidad como una operante relacional jerárquica con el deíctico-Yo. Para ello, en Törneke et al. (2015) hemos propuesto tres estrategias de trabajo en sesión, teniendo en cuenta que ese repertorio se forma a través de conductas dentro y fuera de la sesión. Al lector avezado en la psicoterapia analítica funcional (FAP), no se le escapará reconocer la confluencia de ACT y FAP. Las tres estrategias de trabajo en sesión sumariamente consideradas se indican a continuación y las tres operan para transformar la función de la conducta desde el enmarque jerárquico:

(1) Ayudar al paciente para que llegue a experimentar el contacto con los resultados del patrón problemático (en que resulta de actuar enmarcando en coordinación). Lo que es equivalente al análisis funcional de la regulación problemática en ACT que permite experimentar los pensamientos/emociones/reglas, lo que se hace y para qué se hace, lo que se consigue al hacerlo y lo que se consigue a largo plazo. Es decir, situar el contexto para identificar el verdadero problema y la apertura a su solución.

(2) Ayudar al paciente para que pueda abrir y amplificar las funciones ligadas a lo que tiene sentido personal, a lo que podría entusiasmarle o emocionarle; dicho de otro modo, a las funciones ligadas a estar presente en un contexto de jerarquía, inclusivo en cada momento, de lo que uno siente/piensa/hace.

(3) Ayudar al paciente a producir la habilidad de diferenciarse uno mismo de sus conductas (privadas y públicas) y ejercer la acción de acuerdo a las funciones que surgen o se amplifican (ver punto 2) al enmarcar la propia conducta en un contexto jerárquico.
Las tres estrategias van dirigidas a generar un repertorio de flexibilidad ante la propia conducta tanto en el paciente como en el terapeuta de tal modo que las conductas de paciente y terapeuta interactúan para producir las transformaciones necesarias en la construcción del repertorio de flexibilidad. Su puesta en marcha requiere una comprensión a fondo del modelo y de práctica sistemática.

Presentar ACT unida a la teoría general sobre el lenguaje y la cognición, al papel de las contingencias y a la comprensión de la Teoría del Marco Relacional, es conectar lo básico y las aplicaciones. Es este modo de presentar la terapia contextual donde se encuentra coherencia con la Contextual Behavioral Science (CBS, Hayes, Barnes-Holmes, \&Wilson, 2014). La CBS es un modo de organizar las base filosófica (el Contextualismo Funcional), la investigación sistemática sobre la conducta y la cognición $y$, las aplicaciones efectivas sostenidas por el conocimiento que proporciona la investigación sistemática y sus derivaciones.

Si pensamos en la CBS a modo de un gran árbol (Luciano, 2014), ACT sería una de sus ramas. Sería una de las aplicaciones unidas al tronco que recogería los conocimientos sobre el funcionamiento de la conducta. Ahí hallaríamos un tronco cambiante según los hallazgos, desde el conocimiento sobre cómo operan las contingencias a cómo operan para producir el repertorio relacional -lenguajey, más, cómo opera la transformación de funciones en el repertorio relacional.

Por ser breve, contemplar ACT a modo de una rama de ese gran árbol mostraría a ACT como una terapia no aislada ni estática sino 
flexible en cuanto unida a los avances que se vayan dando en la investigación en lenguaje. Ver ACT de ese modo ofrece una panorámica de la Terapia Contextual en el ámbito de la Behavioral Science (CBS) en la que la ciencia de la conducta se abre a otras ciencias (otros árboles del conocimiento) mostrando un horizonte extraordinario de confluencia que sitúa la conducta humana en el contexto del resto de eventos en la naturaleza.

\section{Concluyendo}

La definición de flexibilidad propuesta es compatible con el trabajo experimental realizado hasta ahora y permite dejar el hexaflex en los límites para los que fue generado. La definición relacional de inflexibilidad iría mucho más allá de lo habido hasta ahora pues trata de englobar todo ello en un marco básico que coordine teoría e investigación con fines de prevención y cambio. $Y$ esto resulta coherente con la visión en la que ACT se incluye, la Ciencia Conductual-Contextual (Contextual Behavioral Science). El interplay entre aplicaciones y leyes que emanan del conocimiento experimental no sólo sirve para nutrirse mutuamente al generar productos útiles en ambos niveles, sino que proporciona fuerza a la disciplina unida a la coherencia y flexibilidad en el conocimiento que ofrece.

En síntesis, situar ACT como una terapia contextual va más allá de los términos y más allá de la propia terapia. ACT es sólo una parte aplicada del conocimiento sobre las leyes que regulan nuestra condición y cuanto más avance este conocimiento más precisas serán las intervenciones. Sin que pudiere ser de otro modo, habrá más escenarios en la permanente evolución de ACT como terapia contextual. Si bien los escenarios están ahí disponibles de modo que los interesados puedan situarse allá donde para cada uno sea menester.

\section{Referencias}

Foody, M., Barnes-Holmes, Y., \& Barnes-Holmes, D. (2012). The role of self in Acceptance and Commitment Therapy (ACT). En L. McHugh, \& I. Stewart (Eds.), The self and perspective taking: Research and applications (pp. 126142). Oakland, CA: New Harbinger.

Foody, M., Barnes-Holmes, Y., Barnes-Holmes, D., \& Luciano, C. (2013). An empirical investigation of hierarchical versus distinction relations in a self-based $A C T$ exercise. International Journal of Psychology and Psychological Therapy, 13, 373-385.

Gil, B., Ruiz, F. J., Valdivia-Salas, S., \& SuárezFalcón, J. C. (2015, julio). The differential effect of defusion exercises based on deictic and hierarchical framing on tolerance experimental task. Comunicación oral presentada en la ACBS World Conference XIII, Berlín.

Hayes, S. C. (1987). A contextual approach to therapeutic change. En N. Jacoboson (Ed.), Psychotherapists in clinical practice: Cognitive and behavioral perspectives (pp. 327-387). New York: Guilford.

Hayes, S. C., Strosahl, K. D., \&Wilson, K. G. (1999). Acceptance and commitment therapy. An experiential approach to behavior change. New York: Guilford Press.

Hayes, S. C., Wilson, K. G., Gifford, E. V., Follette, V. M., \& Strosahl, K. D. (1996). Experiential avoidance and behavioral disorders: A functional dimensional approach to diagnosis and treatment. Journal of Consulting and Clinical Psychology, 64, 11521168.

Hayes, S. C., Barnes-Holmes, D., \& Roche, B. (Eds.) (2001). Relational frame theory. A postSkinnerian account of human language and cognition. New York: Kluwer. 
Hayes, S. C., Barnes-Holmes, D., \& Wilson, K. G. (2012). Contextual behavioral science: Creating a science more adequate to the challenge of the human condition. Journal of Contextual Behavioral Science, 1, 1-16.

Hayes, S. C., Strosahl, K. D., Bunting, K., Towhig, M., \& Wilson, K. (2004). What is Acceptance and Commitment Therapy? En S. C. Hayes, \& K.D.Stroshal (Eds), A practical guide to ACT. Springer, NY.

Lopez, J. C., \& Luciano, C. (2015). Analyzing transformation of functions in deictic and hierarchical context of the own behavior. Comunicación oral presentada en la ACBS World Conference XIII, Berlín.

Luciano, C. (2014). The future of the CBS. Symposium ACBS Dublin. December, 2014. (El árbol como metáfora de la aproximación contextual. IJPPT, en prensa).

Luciano, C. Rodríguez-Valverde, M., \& Gutiérrez-Martínez, O. (2004). A proposal for synthesizing verbal contexts in Experiential Avoidance Disorder and Acceptance and Commitment Therapy. International Journal of Psychology and Psychological Therapy, 4, 377-394.

Luciano, C., Ruiz, F. J., Vizcaíno-Torres, R., Sánchez, V., Gutiérrez-Martínez, O., \& LópezLópez, J.C. (2011). A relational frame analysis of defusion interactions in Acceptance and Commitment Therapy. A preliminary and quasi-experimental study with atrisk adolescents. International Journal of Psychology and Psychological Therapy, 11, 165-182.

Polk, K. L., \& Schoendorff, B. (Eds.) (2014). The ACT Matrix: A new approach to building psychological flexibility across settings and populations. Reno, NV: Context Press.

Ruiz, F. (2010). A review or ACT empirical evidence. Correlational, experimental psychopathology, components and outcome studies. International Journal of Psychology and Psychological Therapy, 10, 125-162.

Stroshal, K., Robinson, P., \& Gustavsson, T. (2014). Brief Interventions for Radical Change. . Oakland, CA: New Harbinger Inc.
Skinner, B. F. (1974). About Behaviorism. New York: Knopf.

Törneke, N. (2015). Aprendiendo TMR: una introducción a la Teoría del Marco Relacional y sus aplicaciones clínicas (Trads. M. Á. Barbero \& E. Moreno Fuentes). Jaén: MICPSY.

Törneke, N., Luciano, C., Barnes-Holmes, Y., \& Bond, F. (2015). Relational Frame Theory and three core strategies in understanding and treating human suffering). En R. D. Zettle, S. C. Hayes, D. Barnes-Holmes, \& A. Biglan (Eds.), The Wiley Handbook of Contextual Behavioral Science (pp. 254-272). WileyBlackwell

Villatte, M., Villatte, J. L., \& Hayes, S. C. (2016). Mastering the clinical conversation: Language as intervention. New York: Guilford Press.

Wilson, K., \& Luciano, C. (2002). Terapia de Aceptación y Compromiso. Madrid: Pirámide. 